\title{
Effect of Selection for Productive Traits in Broiler Male Lines on Embryo Development
}

\section{Author(s)}

Schmidt GS

Figueiredo EAP'

Pesquisadores da Embrapa Suínos e Aves.

Mail Address

GS Schmidt

Embrapa Suínos and Aves, BR 153 - km 110,

Caixa Postal 21

89.700-000. Concórdia, SC, Brazil.

Phone: (0xx49) 441-0400

Fax (0xx49) 441-0497

E-mail: schmidt@cnpsa.embrapa.br.

\section{ABSTRACT}

This experiment aimed at evaluating the effect of genetic selection on embryo development of two male broiler lines ( $L L$ and $L L C$ ). $L L$ line was selected for eight generations for growth, carcass, and reproduction traits. The LLc line was derived from LL line in 1985, and kept under a random mating system with no selection. Fertile eggs were collected and individually weighed for four periods of five consecutive days, with two-week intervals. For every period, a total of 960 eggs/line were identified and separated in four groups of 240 eggs, and then stored for subsequent incubation. At 9 (P9), 11 (P11), 13 (P13), 15 (P15), 17 (P17) and 21 (P21) days of incubation, eggs and embryos were weighed $(\mathrm{EmW})$. Egg weight (EgW) was similar between the two lines. The differences in EmW started to be significant after P15, resulting in a $2.03 \mathrm{~g}$ difference in hatchling weight, indicating genetic changes of $0.54 \%$ by generation, which can be attributed to selection for body weight. The correlation between EgW and EmW started to be significant after P13, and correlations of 0.738 and 0.712 (P21) were observed for $\mathrm{LL}$ and LLC, respectively. Hatchling weight corresponded to 71.94 (LL) and 68.96 (LLC) of EgW. The estimated increases in P21 resulting from each $1.0 \mathrm{~g}$ increase in EgW were $0.73 \mathrm{~g}(\mathrm{LL})$ and $0.69 \mathrm{~g}(\mathrm{LLC})$.

\section{INTRODUCTION}

Embryo development is influenced by several factors that determine the efficiency of incubation and chick quality. Egg physical quality, embryo developmental stage at lay, time spent and prevailing conditions between egg lay and storage, storage and incubation conditions, and incubation time are essential factors to be considered in the production of good quality chicks. The importance of each factor changes with embryo development.

For embryos at the initial development phase, egg size is positively correlated to the number of embryo cells, and negatively correlated with their size (Wiley, 1950). Schmidt et al. (2003a) found a larger number of germ cells in selected lines as compared to control lines.

The correlation between egg weight and embryo weight increases with embryo development stage, and becomes significant after day 13 of incubation. Final chick weight is primarily determined by initial egg weight, typically corresponding to 62 to 78\% (Whiting \& Pesti, 1983; Wilson \& Harms, 1988; Schmidt et al., 2003b), and secondarily by weight loss during incubation, eggshell weight and egg residue weight, genetic line, incubation time and conditions, breeder age, and chick sex.

Individual or genetic line characteristics affect the correlation between egg weight and chick weight. Schmidt et al. (2003b) observed a 3.0\% reduction in that correlation in selected lines as compared to control lines after eight generations of selection, with a reduction of $2.5 \%$ in 
the egg weight to chick weight ratio. Increases in chick weight resulting from an increase of $1.0 \mathrm{~g}$ in egg weight were of 0.71 and $0.68 \mathrm{~g}$ for selected and control lines, respectively.

Embryos from larger eggs may use egg nutrients more efficiently. This hypothesis may be partially explained by the positive correlation between egg weight and the ratio between egg weight and chick weight (Whiting \& Pesti, 1983; Yannakopoulus \& Tserveni-Gousi, 1987; Skewes et al., 1988).

Despite the considerable variation in the results of studies on the effect of chick weight on broiler performance, particularly due to the complexity of controlling all factors that affect the involved characteristics, several studies show a positive correlation with market weight, mortality, and feed conversion ratio (Hearn, 1986).

The objective of the present study was to estimate the effect of the selection for productive traits in male broiler genetic lines on embryo development.

\section{MATERIAL AND METHODS}

Two experimental male broiler genetic lines ( $\mathrm{LL}$ and LLc) kept by the broiler genetic improvement program of Embrapa Suínos and Aves, located in Concórdia, SC, Brazil were used. LL line was selected for growth (body weight, feed intake, and feed conversion ratio), carcass (carcass, breast, and thigh yields), and reproductive (fertility and hatchability) traits for eight generations. The control line (LLC) derived from $L L$ in 1985, and was maintained under a random mating system with no selection. Therefore, both lines had the same genetic background, and their differences are attributed to selection.

In 2004, a group of 34-week-old 300 females and 30 males of each line was housed in individual cages. Artificial insemination was carried out at a 1:10 male to female ratio. Fertile eggs were collected during four periods of five consecutive days each with two-week intervals, allowing four incubations.

A total number of 960 eggs per line per collection period was individually weighed, identified, and separated in four groups of 240 birds each according to weight. Eggs were then stored in an egg-storage chamber, with temperature $\left(14^{\circ} \mathrm{C}\right)$ and humidity $(78 \%)$ control. Eggs were transferred to the pre-heating room eight hours before incubation before setting.

The live embryos of 160 eggs of four replicates of 40 eggs incubated for each development period were removed on days 9 (P9), 11 (P11), 13 (P13), 15 (P15), and 17 (P17) of incubation. After being washed and dried, embryos were individually weighed. On day 18, the remaining eggs were transferred to a hatcher and placed in individual compartments, where they remained until hatching, when the chicks were individually weighed (P21).

The parameters egg weight and embryo weigh were analyzed according to the following mathematical model:

$Y_{j k l m}=+L_{j}+I_{k}+L I_{j k}+A_{1}+L A_{j l}+I A_{k j}+L I A_{j k l}+E j_{k l m}$

where: $Y_{j \mathrm{klm}}$ is the analyzed $Y$ parameter; estimated population mean; $L_{j}$, genetic line effect $j(j=$ and and $2) ; I_{k^{\prime}}$ incubation effect $k(k=1,2,3$, and 4$) ; L_{j k^{\prime}}$ effect of the interaction line * incubation; $A_{1}$, effect of embryo age $\left(A=9,11,13,15,17\right.$, and 21); $L A_{j \mid}$ effect of the interaction incubation * age; $\mid A_{k j}$, effect of the interaction incubation * age; $L I A_{j k \mid}$, effect of the interaction line * incubation * age; and $\mathrm{Eij}_{\mathrm{klm}}$, random error.

The deviations between the selected (LL) and the control (LLC) lines were used as indicators of the genetic changes resulting from artificial selection. The correlation between egg weight and embryo weight was determined, and embryo development curve as a function of incubation time was calculated by analysis of regression.

Analyses were carried out using the statistical procedures of SAS (SAS, 1996) software package, and means were compared by the test of Tukey.

\section{RESULTS AND DISCUSSION}

The analysis of variance and the means obtained for egg weight (EgW) and embryo weight (EmW) for the different incubation periods are presented in Tables 1 and 2 , respectively.

The effects of line, incubation, age, and their respective interactions on EgW were not significant (Table 1). The lack of differences in egg weight between LL (68.30g) and LLC (68.00g) indicates that the objective of selection of maintaining the same egg average weight during eight generations was effective.

The differences in embryo development between LL and LLC became significant after P15 (Figure 1), resulting in a $2.03 \mathrm{~g}$ difference in chick weight at hatch. Considering eight generations of selection, a genetic gain of $0.54 \%(0.25 \mathrm{~g})$ per generation is estimated. As EgW remained similar between the lines, which had 
the same genetic background, the change in EmW (Figure 2) may be considered an indirect response to the selection for body weight. Schmidt et al. (2003) found similar results with female genetic lines, which selection for body weight was not strongly emphasized, obtained a $1.79 \mathrm{~g}$ difference in hatchling weight between the selected and the control lines. When the results of the present experiment are compared with the finding of Schmidt et al. (2003), differences between male and females lines of $1.42 \mathrm{~g}$ and $1.36 \mathrm{~g}$ were determined for the selected and the control lines, respectively. The higher number of skeletal-muscle germ cells (somites) during the initial stage of embryo development in lines selected for body weight may partially explain the variation in EmW (Schmidt et al., 2003a). Differences in the chemical composition and/ or in the efficiency on nutrient utilization by the embryo may explain the other part of variation (Whiting \& Pesti, 1983; Yannakopoulos \& Tserveni-Gousi, 1987).

Table 1 - Mean square (MS) and coefficient of variation (CV) of egg weight (EgW) and embryo weight (EmW).

\begin{tabular}{lcrc}
\hline \multirow{2}{*}{ Variation source } & Degrees of freedom & \multicolumn{2}{c}{ MS } \\
\cline { 3 - 4 } & & EgW & EmW \\
\hline Line $(L)$ & 1 & 3.1965 & $28.6520^{*}$ \\
Incubation $(I)$ & 3 & 2.6322 & 0.0621 \\
$L^{*} \mid$ & 3 & 1.5926 & 0.3085 \\
Age $(A)$ & 5 & 1.5261 & $9.7075^{*}$ \\
$L^{*} A$ & 5 & 0.8119 & 8.3599 \\
I*A $^{*}$ & 15 & 0.9925 & 0.7052 \\
* $^{*} A$ & 15 & 1.7840 & 1.050 \\
Residue & 148 & 2.0313 & 0.4533 \\
CV $(\%)$ & & 2.96 & 4.25 \\
\hline
\end{tabular}

$*(p<0.01)$.

Table 2 - Egg weight (EgW) and embryo weight (EmW) means of the $\mathrm{LL}$ and $\mathrm{LLC}$ lines during different incubation periods.

\begin{tabular}{lccccc}
\hline \multirow{2}{*}{ Embryo age } & \multicolumn{2}{c}{ EgW $\mathbf{( g )}$} & & \multicolumn{2}{c}{ EmW (g) } \\
\cline { 2 - 3 } \cline { 5 - 6 } & LL & LLC & & LL & LLc \\
\hline 9 & 68.29 & 68.06 & & $2.36 \mathrm{a}$ & $2.37 \mathrm{a}$ \\
11 & 67.77 & 67.41 & & $4.91 \mathrm{a}$ & $5.07 \mathrm{a}$ \\
13 & 68.76 & 67.96 & & $10.14 \mathrm{a}$ & $10.26 \mathrm{a}$ \\
15 & 68.10 & 68.42 & & $19.05 \mathrm{a}$ & $18.07 \mathrm{~b}$ \\
17 & 67.89 & 67.89 & & $29.56 \mathrm{a}$ & $27.71 \mathrm{~b}$ \\
21 & 68.98 & 68.98 & & $48.91 \mathrm{a}$ & $46.88 \mathrm{~b}$ \\
Mean & 68.30 & 68.00 & & - & - \\
\hline
\end{tabular}

Means followed by different letters within the same trait and age are significantly different $(p<0.01)$.

Changes in EmW resulting from selection changed EmW correlation with EgW (Table 3), with significant differences after P13. The correlations of 0.261 and 0.231 (P13); 0.292 and 0.282 (P15); 0.351 and 0.319 (P17); and 0.738 and 0.712 (P21), for LL and LLC, respectively, were similar to those obtained by Schmidt et al. (2003b) for female lines, and lower than those verified by Hassan \& Nordskog (1971), who estimated correlations closer to zero at P14, 0.50 at P16, and 0.90 at P21. The stronger emphasis on the selection for body weight in the male line, as compared to the female line (Schmidt et al., 2003b) increased in 1.4\% the correlation between EgW and hatchling weight. In general, the range found in literature for P21 is between 0.50 and 0.95 (Yannakopoulos \& TserveniGousi, 1987).

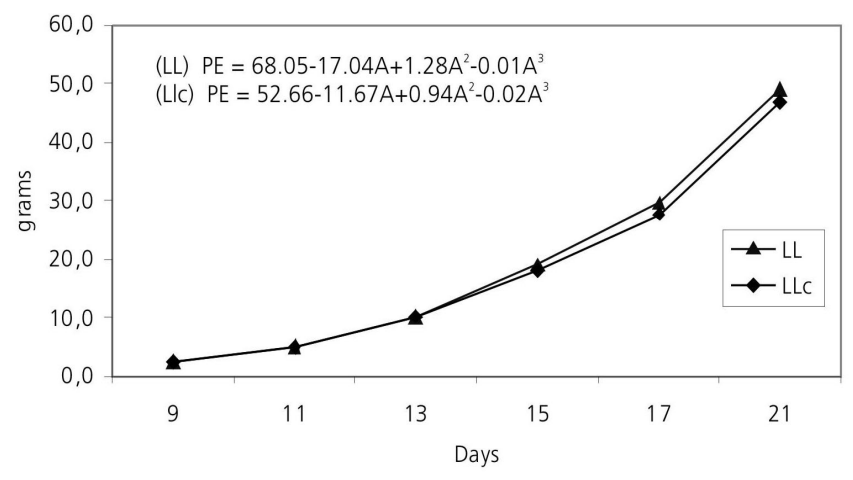

Figure 1 - Embryo development curves as a function of incubation period.

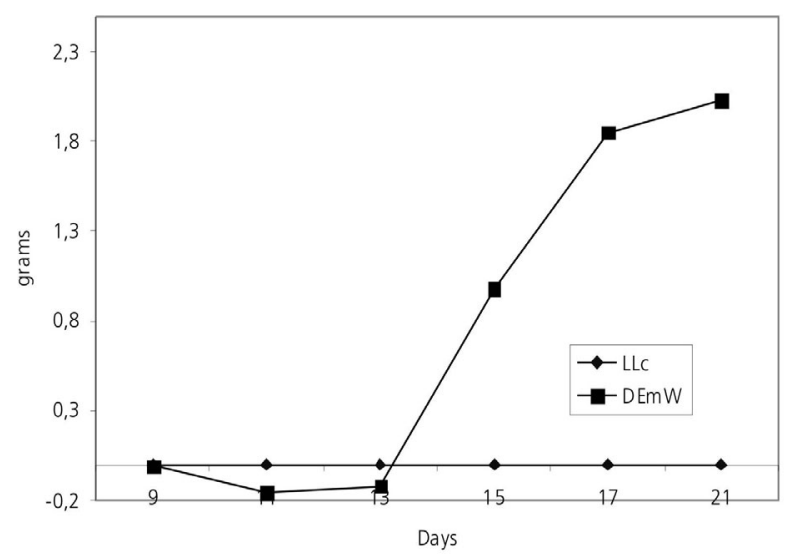

Figura 2 - Embryo weight deviation (DEmW) between the selected and the control lines in the different development stages.

Hatchling weight corresponded to 71.94 and $68.96 \%$ of the egg weight (Table 3 ) for LL and LLC, respectively, which is consistent with the findings of Schmidt et al. (2003b). After P13, increases in embryo weight for each $1.0 \mathrm{~g}$ increase in EgW were 0.16 (P13), 0.29 (P15), 0.45 (P17), and 0.73g (P21) for the LL line, and 0.15 (P13), 0.27 (P15), 0.41 (P17), and 0.69g (P21) for LLC. The selection for body weight increased the correlation between EgW and P21 in $4.32 \%$. Genetic differences in chick weight to egg weight were 
Table 3 - Estimated percentages of embryo weight relative to egg weight (EmW\%), correlation between egg weight and embryo weight $(r)$, effect of the increase of $1.0 \mathrm{~g}$ in egg weight on hatchling weight (DF), and embryo weight deviation (DV) between LL and LLc lines in the different incubation periods.

\begin{tabular}{|c|c|c|c|c|c|c|c|}
\hline \multirow[t]{2}{*}{ Age } & \multicolumn{3}{|c|}{ LL } & \multicolumn{3}{|c|}{ LLC } & \multirow[t]{2}{*}{ DV } \\
\hline & EmW(\%) & $r$ & DF & EmW (\%) & $r$ & $\overline{\mathrm{DF}}$ & \\
\hline 9 & 3.50 & 0.109 & 0.03 & 3.53 & 0.10 & 0.04 & -0.01 \\
\hline 11 & 7.35 & 0.172 & 0.07 & 7.63 & 0.151 & 0.08 & -0.16 \\
\hline 13 & 14.96 & 0.261 * & 0.16 & 15.32 & $0.231 *$ & 0.15 & -0.12 \\
\hline 15 & 28.39 & 0.292 * & 0.29 & 26.80 & 0.282 * & 0.27 & 0.98 \\
\hline 17 & 44.19 & 0.351 * & 0.45 & 41.43 & $0.319 *$ & 0.41 & 1.85 \\
\hline 21 & 71.94 & $0.738 *$ & 0.73 & 68.96 & 0.712 * & 0.69 & 2.03 \\
\hline
\end{tabular}

$r$ values followed by * are significantly different $(p<0.01)$.

observed by Whiting \& Pesti (1983), who reported that dwarf broiler lines presented a lower ratio $(63.7 \%)$ as compared to non-dwarf lines (68.4\%).

Embryo development curve differences between the selected and the control lines are significant after 15 days of embryo age (Figure 2). The analysis of regression indicates that the cubic response explains 95\% of the variation in embryo development (Figure 1).

\section{CONCLUSIONS}

The selection for body weight resulted in an embryo development response after 13 days of incubation, with an estimated genetic gain of $0.54 \%$ in hatchling weight per generation. The correlation between egg weight and embryo weight increased with incubation time, and was the highest at the final third of incubation.

\section{REFERENCES}

Hassan EM, Nordskog AW. Effect of egg size and heterosis on embryonic growth and hatching speed. Genetics 1971; 67:279285.

Hearn PJ. Making use of small hatching eggs in an integrated broiler company. British Poultry Science 1986; 27:498-504.
SAS statistical analysis systems user's guide: Stat. Version 6. 4th ed. Cary: SAS Institute; 1996. v.2.

Schmidt GS, Coutinho LL, Figueiredo EAP. et al. Morphological genetic markers for selection on broiler. Brazilian Journal of Poultry Science 2003a; 5(3):175-178.

Schmidt GS, Figueiredo EAP, Ledur MC, Alves HJ. Effect of selection for productive traits in broiler maternal lines on embryo development. Brazilian Journal of Poultry Science 2003b; 5(2):125129.

Skewes PA, Wilson HR, Mather FB. Correlation among egg weight, chick weight, and yolk sac weight in Bobwhite quail (Colinus virginianus). Florida Scientist 1988; (51):159-162.

Whiting TS, Pesti GM. Effects of Dwarfing gene (dw) on egg weight, chick weight, and chick weight:egg weight ratio in a commercial broiler strain. Poultry Science 1983; 62:2297-2302.

Wiley WK. The influence of egg weight on the pre-hatching and post-hatching growth rate in the fowl. II. Egg weight-chick weight ratios. Poultry Science 1950; 29:595-604.

Wilson HR, Harms RH. Chick weight varies directly with egg weight. Poultry-Misset International 1988; 4:10-13.

Yannakopoulos AL, Tserveni-Gousi AS. Relationship of parent's age, hatching egg weight and shell quality to day-old chick weight as influence by oviposition time. Poultry Science 1987; 66:829833. 